\title{
Ensino do Pensamento Computacional como Estratégia na Regulação Emocional de Alunos: Um Mapeamento Sistemático da Literatura
}

\author{
Geisiane de Souza Matos ${ }^{1}$, Sara M. Silva ${ }^{1}$, Sandy Hiorrana L. Farias ${ }^{2}$, \\ Fabíola Pantoja O. Araújo ${ }^{1}$, Josivaldo S. Araújo ${ }^{1}$
}

\author{
${ }^{1}$ Instituto de Ciências Exatas e Naturais (ICEN) - Universidade Federal do Pará (UFPA) \\ Telefone 3201-7405 - 66075-110 - Belém - PA - Brasil \\ ${ }^{2}$ Instituto de Tecnologia (ITEC)- Universidade Federal do Para (UFPA) \\ Telefone 3201-7250 - 66075-900 - Belém - PA - Brasil
}

geisi.mattos.s, maru.merces18, sandylimafarias15\{@gmail.com\}, fpoliveira, josivaldo\{@ufpa.br\}

\begin{abstract}
Computational thinking is widely recognized as a fundamental skill for everyone, and even more important when added to teaching, be it from basic education to higher education. To analyze research, methodologies and software that already exist, this work presents a Systematic Mapping of Literature that aims to investigate the use of computational thinking as a strategy to develop students' emotional skills, computational thinking being a means of awakening cognitive abilities in the intellectual and emotional field. The searches were carried out in national and international databases, in the period from 2016 to 2020. In return, 65 studies were obtained, of which 10 were selected after the filtering steps determined in the research protocol.
\end{abstract}

Resumo. O Pensamento Computacional é amplamente reconhecido como uma habilidade fundamental para todos e ainda mais importante quando agregado ao ensino, da educação básica ao ensino superior. Com o propósito de analisar pesquisas, metodologias e softwares que já existem, este trabalho apresenta um Mapeamento Sistemático da Literatura que visa investigar o uso do Pensamento Computacional como estratégia para desenvolver as habilidades emocionais dos alunos, sendo o pensamento computacional um meio de despertar habilidades cognitivas no campo intelectual e emocional. As buscas foram realizadas em bases de dados nacionais e internacionais, no período de 2016 à 2020. Como retorno obteve-se 65 estudos, dos quais 10 foram selecionadas após as etapas de filtragem determinadas no protocolo de pesquisa.

\section{Introdução}

O cenário dos dias atuais demonstram que, com o passar dos tempos, nos conectamos mais às tecnologias. As Novas Tecnologias de Informação e Comunicação (NTICs) seguem influenciando mudanças nas formas de comunicação e educação [Lima et al. 2020]. Apesar dessa ampla conectividade, a maioria das redes de ensino ainda não possuem em seus currículos o ensino de disciplinas que envolvam a Computação como ferramenta para a resolução de problemas. Segundo o Currículo de Referência em Tecnologia e Computação (CRTC), o Pensamento Computacional (PC) tem sido considerado como 
uma das habilidades fundamentais do ser humano, ao lado da leitura, escrita e aritmética [CIEB 2021].

De acordo com as diretrizes da Sociedade Brasileira de Computação (SBC), os fundamentos da Ciência da Computação são bases para o PC, o qual tem por objetivo desenvolver habilidades que envolvam analisar, definir, comparar, solucionar e automatizar problemas de forma sistêmica, por meio da construção de algoritmos, fazendo uso ou não de ferramentas computacionais [SBC 2018]. Inúmeros estudos vêm sendo desenvolvidos, com o objetivo de ampliar o ensino do PC combinado a assuntos do ensino regular. [Lopes et al. 2020].

Outro outro ponto de destaque na educação é a inteligência emocional, sendo, sua importância na aprendizagem, crucial no processo de interação social. Fatores emocionais preparam e orientam comportamentos, sejam em experiências positivas ou negativas [Fonseca 2016]. Os seres humanos têm a capacidade de empregar fatores emocionais em atividades cotidianas, essas emoções podem estar ainda mais presentes e desorganizadas no ambiente escolar [Tessaro and Lampert 2019]. Trabalhar o processo de autoregulação emocional no âmbito escolar, tem o objetivo de auxiliar os alunos a obterem comportamentos e competências dirigidas em processo consciente, possibilitando a capacidade de empatia, trabalho em equipe e maior desempenho dos níveis de aprendizado [Rodrigues 2015]. A regulação emocional é constituida pela capacidade do indivíduo em lidar com situaçãoes de extresse e desafiadoras de forma saudável e eficiente.

Desta forma, o objetivo deste trabalho é realizar um Mapeamento Sistemático da Literatura (MSL), identificando estudos que utilizaram os fundamentos da computação, especificamente do PC como estratégia no processo de regulação emocional de alunos da educação básica ao ensino superior, em ambiente escolar. Com este estudo, pretende-se identicar metodologias, técnicas, softwares ou ferramentas computacionais utilizadas no processo de detecção ou estímulo das habilidades emocionais.

Este artigo segue organizado da seguinte forma: na Seção 2 são apresentados os procedimentos metodologicos desenvolvidos no Mapeamento Sistemáticos da Literatura; na Seção 3 são abordados os resultados aferidos no processo de extração de dados; e, por fim, na Seção 4 são descritas as considerações finais e os trabalhos futuros propostos.

\section{Mapeamento Sistemático da Literatura (MSL)}

Este estudo apresenta um Mapeamento Sistemático da Literatura (MSL), os procedimentos metodologicos percorridos aqui, são baseados na metodologia apresentada na pesquisa de Cabrejos et al. (2018), que apresenta as etapas de execução de estudos secundários realizados na área de informática na educação para ser usado como base na condução de futuros estudos.

O principal objetivo de um MSL é fornecer uma visão geral e formativa de determinada área, sendo utilizado quando há uma necessidade de classificar um determinado campo de pesquisa, organizando tópicos de interesse do estudo [Moher et al. 2015]. Portanto, o MSL faz uso de estudos anteriormente publicados e validados em periódicos e conferências propícios ao conteúdo de pesquisa, sendo estes denominados de estudos primários.

Para condução do Mapeamento, foram seguidos cinco passos propostos por $\mathrm{Ca}$ - 
brejos et al. (2018) sendo eles: (I) definição de questão de pesquisa, (II) definição do protocolo de pesquisa, (III) busca de estudos primários nas bases de dados, (IV) triagem dos estudos pré-selecionados, (V) extração de dados e processo de mapeamento.

Diante desse exposto, a questão formulada para esta pesquisa foi organizada e estrututurada nos critérios recomendados por Keele (2007) sendo: população, intervenção, comparação, resultados e contexto (PICOC). Porém, foram utilizados somente os itens população $(\mathrm{P})$, intervenção $(\mathrm{I})$, contexto $(\mathrm{C})$ e resultados $(\mathrm{O})$ para a construção da questão de pesquisa. $\mathrm{O}$ elemento comparação $(\mathrm{C})$ não se aplica a esta pesquisa por não ser uma Revisão Sistemática da Literatura (RSL).

Nas seções seguintes são discutidas as etapas de planejamento, execução e análise de resultados de acordo com o protocolo previamente estabelecido.

\subsection{Procedimentos Metodológicos}

O processo de um MSL visa identificar e analizar possíveis estudos capazes de responder a questão de pesquisa principal $(\mathrm{QP})$ e as questões específicas (QE) determinadas no protocolo de pesquisa [Paviani et al. 2018]. O protocolo de pesquisa é composto pela definição da questão de pesquisa, String de busca, seleção das bases de dados e critérios de inclusão e exclusão.

O protocolo de busca definido no mapeamento sistemático visa responder a seguinte questão de pesquisa:

- (QP1) "Quais metodologias, técnicas e softwares vêm sendo utilizados no desenvolvimento do Pensamento Computacional de alunos da educação básica ao ensino superior com o objetivo de estimular as habilidades emocionais dos alunos?"

Para obter os resultados esperados, por meio do MSL, além da questão de pesquisa principal, é necessário um conjunto de questões secundárias, as quais serão respondidas durante a fase de extração de informações. As questões secundárias têm o objetivo de esclarecer detalhes importantes que o MSL visa identificar, contribuindo na análise dos estudos. Desta forma, foram elaboradas as seguintes questões específicas:

- (QE1)Quais abordagens computacionais os educadores vêm utilizando na regulação emocional dos alunos?

- (QE2)Quais os efeitos emocionais observados nos alunos com a introdução de atividades que envolvem computação?

- (QE3)Quais emoções têm sido consideradas nos estudos?

- (QE4)Em quais disciplinas a regulagem emocional vêm sendo empregada?

- (QE5)Quais as ferramentas estão sendo utilizadas no processo de regulação emocional dos alunos? Estão disponíveis no mercado ou foram desenvolvidas especificamente para a aplicação do trabalho?

\subsection{String de Busca}

A String de busca definida no protocolo de pesquisa teve sua apresentação em duas variações. As adaptações na String foram necessárias pelo fato da pesquisa decorrer em bases de dados nacionais e internacionais. A seguir, na Tabela 1, está a descrição das Strings. 
Tabela 1. Strings de busca versão Português (Brasil) e Inglês.

\begin{tabular}{|c|}
\hline Strings de Busca \\
\hline (("regulação da emoção"OR "regulação emocional" \\
OR "autorregulação"OR "inteligência emocional"OR "habilidades emocionais") \\
AND (aprendizagem OR "ensino"OR educação) \\
AND ("computação"OR "pensamento computacional")) \\
(("emotion regulation"OR "emotional regulation" \\
OR "self-regulation"OR "emotional intelligence"OR "emotional skills") \\
AND (learning OR "teaching"OR education) \\
AND ("computing"OR "computational thinking")) \\
\hline
\end{tabular}

\subsection{Processo de Busca}

Como exposto anteriormente, as questões de pesquisa (principal e específicas) têm como objetivo identificar estudos que abordam o Pensamento Computacional e a regulação emocional aplicado à educação.

Para responder as questões levantadas nesse trabalho, foram selecionados estudos publicados entre janeiro de 2016 e agosto de 2020. Este intervalo foi estabelecido, após a identificação da disseminação da importância do desenvolvimento de habilidades emocionais no ensino, com o acontecimento do "Fórum Internacional de Políticas Públicas"em março de 2014, nesta ocasião foram discutidas por inúmeros países a importância do desenvolvimento emocional na prática pedagógica, assim como a utilização de múltiplas linguagens e diferentes estilos para atingir as diferentes inteligências, sendo este evento a linha condutora para as tendências atuais acerca do tema [Abed 2016].

Foi decidido que seriam considerados estudos publicados nos indiomas Português e Inglês, portanto, foram selecionadas as seguintes bases de dados: ACM Digital Library, Springer Link, IEEExplore Digital Library, Science Direct, Scopus, Simpósio Brasileiro de Informática na Educação e Revista Novas Tecnologias na Educação.

As bases de pesquisas nacionais utilizadas na busca inicial são veículos de publicações na área de Informática na Educação, foi possível identificar que a quantidade de produções brasileiras acerca do tema ainda é escassa. Por outro lado, as bases internacionais retornaram um quantitativo maior. Optou-se por utilizar a mesma String de busca, diferenciando-se apenas os idiomas. As Strings de busca foram aplicadas na pesquisa automática gerida pelas bases, e também foram realizadas buscas manuais com os palavras chaves definidas na String. O gráfico da Figura 1 apresenta o total de publicações retornadas de cada uma das bases pesquisadas, representando um total de 65 trabalhos.

\subsection{Critérios de Inclusão e Exclusão}

Os critérios de inclusão e exclusão determinam a aceitação ou não de um estudo primário e são baseados na questão de pesquisa, sendo eles responsáveis por filtrar os trabalhos, criando uma lista de possíveis artigos primários aceitos e outra com artigos excluídos. Para a inclusão de um estudo é necessário que o estudo se enquadre em ao menos um critério de inclusão e que não pertença a nem um critério de exclusão.

Após as pesquisas automáticas e manual com a String de busca nas bases, foi realizada uma filtragem inicial por meio da leitura do título, resumo e palavras-chaves 


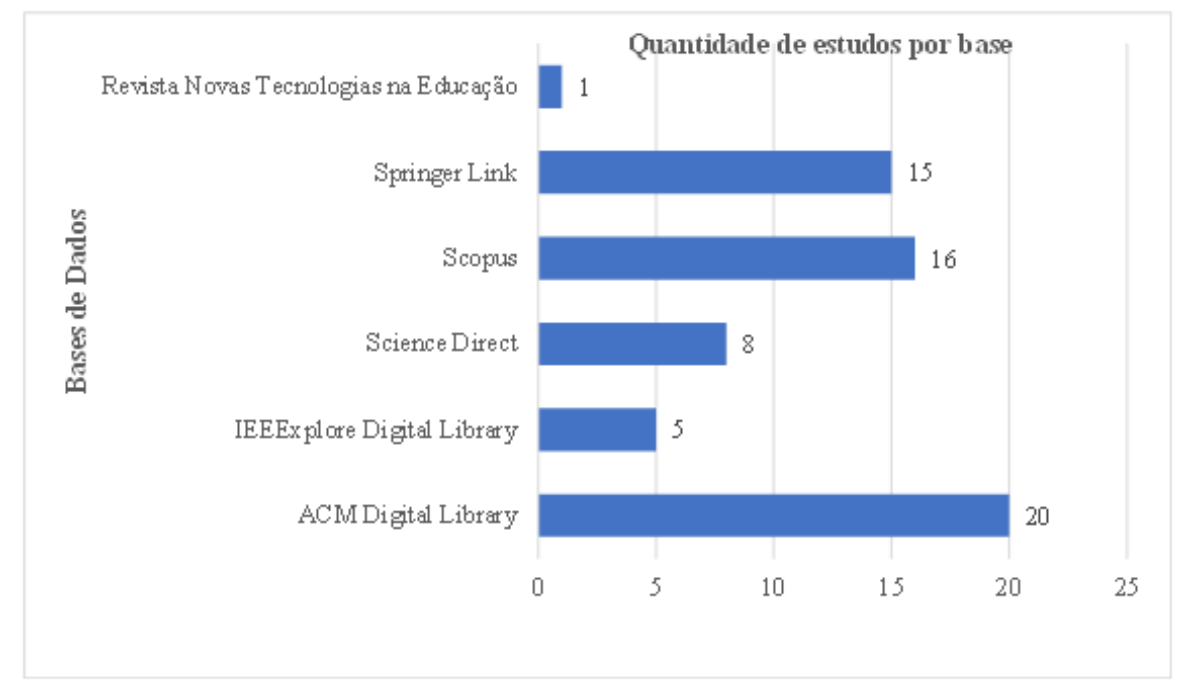

Figura 1. Gráfico da quantidade de estudos por base.

dos artigos retornados, com o objetivo de eliminar trabalhos duplicados. Inicialmente foram retornados 65 estudos e, após essa etapa de análise, restaram 64 trabalhos.

Posteriormente, iniciou-se a filtragem dos estudos a partir dos critérios de inclusão (CI) e criterios exclusão (CE).

Nessa atividade, dois autores aplicaram os CI e CE, individualmente a cada trabalho selecionado na filtragem inicial, em caso de divergências um terceiro autor era acionado a aplicar os critérios no estudo. Os resultados obtidos sob análise individual dos autores foram confrontados e comparados. Os critérios de inclusão aplicados nesse MSL estão descritos a seguir.

- CI01 - A pesquisa aborda o uso da computação como ferramenta no estímulo de habilidades emocionais.

- CI02 - A pesquisa é aplicada na educação.

- CI03 - A pesquisa é um estudo primário.

- CI04 - O público da pesquisa são alunos e professores da rede de ensino básica e/ou superior.

- CI05 - As publicações são de 2016 à 2020.

- CI06 - Ser em português ou inglês.

Os critérios de exclusão tem por objetivo tratar trabalhos inacessíveis, em idiomas que não sejam os definidos no protocolo de pesquisa e estudos que não se enquadrão na questão principal. Os critérios utilizados para excluir trabalhos inválidos a essa pesquisa foram:

- CE01 - A pesquisa não é aplicada na educação.

- CE02 - A publicação é um artigo resumido.

- CE03 - A publicação é um estudo secundário (MSL/RSL).

- CE04 - A pesquisa não está escrita em português ou inglês.

- CE05 - Ser de base de dados particular.

- CE06 - Ter sua publicação anterior a 2016.

- CE07 - A pesquisa não aborda a regulagem emocional de alunos. 
A Figura 2, apresenta o número de trabalhos aceitos após a aplicação dos critérios de inclusão e exclusão.

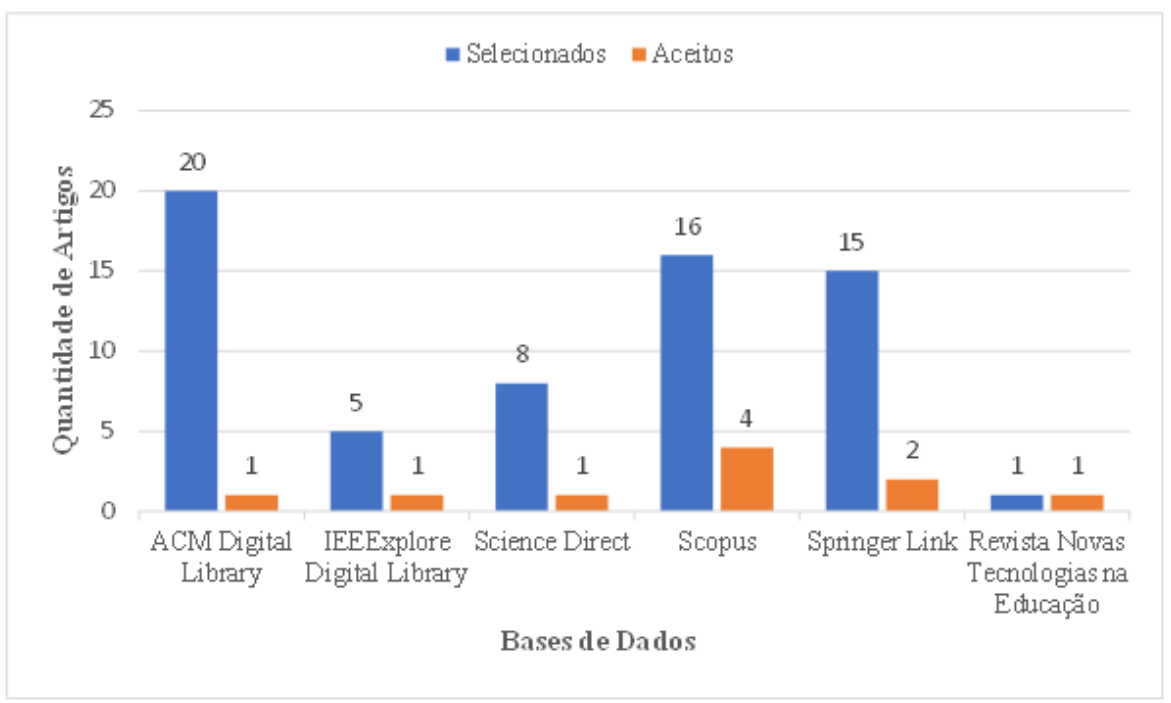

Figura 2. Artigos aceitos por base.

\section{Resultados}

Nesta seção estão expostas as respostas para a QP e as demais QE. Essas respostas são o resultado da análise e extração de dados dos 10 estudos primários aceitos no MSL.

\subsection{Extração de Dados}

$\mathrm{Na}$ etapa de extração de dados é realizada a organização dos dados extraídos, podendo ser feita em formato de relatórios ou gráficos, com o objetivo de registrar detalhes de referências e informações que tratam as questões de pesquisa. Na Tabela 2 são apresentados os títulos resumidos dos estudos, seus respectivos autores e ano de publicação.

Tabela 2. Estudos Primários Analisados

\begin{tabular}{|c|l|l|}
\hline Código & Título Resumido & Referência \\
\hline E01 & Perspectives for effective integration of e-learning & [Akugizibwe et al. 2019] \\
\hline E02 & Matching self-reports with electrodermal activity & [Dindar et al. 2019] \\
\hline E03 & Students' acceptance and readiness for E-learning & [Ngampornchai 2016] \\
\hline E04 & Socioemotional Skill Domains in Robotics & [Arevalo et al. 2020] \\
\hline E05 & Strengths against psychopathology in adolescents & [Piqueras et al. 2019] \\
\hline E06 & Toward effective robot-child tutoring & [Ramachandran et al. 2019] \\
\hline E07 & An ISMP Approach for Promoting Design Innovation & [Chen et al. 2020] \\
\hline E08 & An instrument to assess self-efficacy & [Danielsiek et al. 2018] \\
\hline E09 & CADAP - Uma Ferramenta de apoio & [Paxiuba et al. 2016] \\
\hline E10 & A Computation Model for Learning Programming & [Rafique et al. 2017] \\
\hline
\end{tabular}

\subsection{Respostas às Questões de Pesquisa}

Aqui nesta seção, serão apresentadas as respostas da questão principal formulada no protocolo de pesquisa deste MSL. Através dessas respostas, busca-se investigar as técnicas, 
métodos, softwares ou ferramentas computacionais que vêm sendo utilizadas no processo de estímulo da regulação emocional de alunos. Em seguida, serão respondidas as questões específicas, considerando a nomeclatura dos autores disponível na Tabela 2, disponível de forma detalahada em: https://urless.in/nfxVr.

- (QP1) “Quais metodologias, técnicas e softwares vêm sendo utilizados no desenvolvimento do Pensamento Computacional de alunos da educação básica ao ensino superior com o objetivo de estimular as habilidades emocionais dos alunos?"

$\mathrm{Na}$ fase de extração de dados, os estudos primários foram analisados, sendo identificado que os pesquisadores têm empregado metodologias de ensino aliadas ao uso da Computação e os principais meios são jogos, róbotica, temáticas que envolvem lógica de programação e aplicação de métodos que abordam a Computação Desplugada [E04, E05, E06, E10]. No quesito desenvolvimento de software, os pesquisadores demonstram maior interesse em utilizar ferramentas prontas, gratuitas e disponíveis no mercado. Somente dois estudos apresentaram o desenvolvimento de uma ferramenta [E04, E06], que serão descritas nos resultados da QE05. Outra característa importante identificada é o fato da maioria dos trabalhos [E02, E02, E04, E06, E08, E10] serem desenvolvidos em paralelo à disciplinas de exatas: matemática, física, algoritmos e lógica de programação.

- QE01 "Quais abordagens computacionais os educadores vêm utilizando na regulação emocional dos alunos?

De acordo com as informações extraídas dos estudos primários, os educadores estão aliando ferramentas computacionais à regulação emocional principalmente através do uso de softwares, robôs interativos e atividades virtuais, as quais fazem uso do computador apenas para acesso à internet. Além disso, no trabalho [E05], foi utilizado um software aplicativo apenas para fins avaliativos dos resultados de um estudo dirigido por meio de questionários manuais. No trabalho [E04] foi utilizado um modelo de projeto quase- experimental onde foi desenvolvido um protótipo de robô para ser implementado pelos alunos, seguido da aplicação de dois questionários pré e pós teste que seguiram regras do design thinking voltados para o desenvolvimento das habilidades emocionais dos alunos.

- QE02 "Quais os efeitos emocionais observados nos alunos com a introdução de atividades que envolvem computação?"

Observou-se nos estudos, especificamente em [E03], [E05], [E06], [E07], [E08], [E09], [E10] efeitos satisfatórios no uso da Computação como ferramenta de estímulo das habilidades emocionais dos alunos, sendo destacado nos estudos a capacidade de gerenciar emoções, alcançar objetivos, demostração de empatia, gerenciar relações de forma positiva e tomar decisões de maneira responsável. Também foi identificado que, os alunos desenvolveram maior capacidade de resolução de problemas, maior motivação em experimentar novos problemas e uma maior capacidade de autorregulação individual da aprendizagem, que é a forma como o aluno conduz seu processo de aprendizagem. Além disso, notou-se que o nível de aprendizagem e aceitação dos conteúdos de ensino melhoram quando se têm uma regulação emocional efetiva,sendo esse processo de identificação e comparação de parâmetros realizado por coleta de dados via formulário pré e pós atividades, avaliação emocional por detecção de expressões faciais e também por avaliação documental de turmas anteriores, onde é relacionado um grupo de controle e um grupo de teste. 
No estudo [E07], realizado com 86 alunos do curso de graduação de Engenharia Mecânica, verificou-se que a inteligência emocional associada ao PC pode facilitar o aprimoramento da capacidade de inovação de design no curso de Engenharia. Entretanto, no estudo [E03] realizado com alunos na faixa etária de 18 a 23 anos, mostrou-se pouco efetivo o uso de ferramentas computacionais no auxilio da autorregulação emocional, apenas os alunos mais novos, que eram familiarizados com tecnologias, obtiveram melhores níveis de regulação emocional.

- QE03 "Quais emoções têm sido consideradas nos estudos?"

Dentre as sete emoções básicas propostas por Ekman and Friesen (2003), as principais abordadas nos estudos são raiva, alegria, medo e tristeza. Além destas, trabalhou-se em cima de diversas habilidades socioemocionais, tais como: capacidade de concentração, autorregulação do aprendizado, trabalho em grupo, domínio no desempenho de tarefas, influência social e resiliência.

- QE04 "Em quais disciplinas a regulagem emocional vêm sendo empregada?”

A regulação emocional foi aplicada de maneira interdisciplinar em quatro estudos [E03], [E04],[E05], [E09] seguido da matemática, que foi empregada em dois estudos [E01] e [E06]. Nos demais, teve-se a aplicação em formato livre de ensino, cujo foco foi no ambiente escolar como um todo. Apenas no estudo [E09] é apresentado um software, o qual é capaz de identificar as reações emocionais dos alunos diante de uma aula expositiva, que pode ser usado pelos educadores nas mais diversas disciplinas.

- QE05 "Quais as ferramentas estão sendo utilizadas no processo de regulação emocional dos alunos? Estão disponíveis no mercado ou foram desenvolvidas especificamente para a aplicação do trabalho?"

As ferramentas e softwares utilizados nos 10 artigos selecionados, são listados a seguir.

No trabalho [E01], apresentou-se o uso do software Xerte (XOT), que é um programa gratuito e de uso aberto cujo objetivo é disponibilizar uma plataforma com ferramentas baseadas em navegador que permite que qualquer pessoa com um navegador Web crie materiais de aprendizagem interativos de forma rápida e fácil. Usou-se também a Calculadora Matrix no cálculo de matrizes e o ambiente $\mathrm{R}$ para a criação de gráficos interativos. .

No trabalho de [E05], foi utilizado o programa SPSS PROCESS, que é um software aplicativo do tipo científico. Este software fornece apoio a tomada de decisão que inclui: aplicação analítica, mineração de dados, mineração de texto e estatística que transformam os dados em informações importantes que proporcionam reduzir custos e aumentar a lucratividade. Além deste, fez-se o uso também de questionários manuais: Medida de aceitação e atenção das crianças (CAMM), Questionário de cultura (SDQ), Questionário de Inteligência Emocional Traço - Formulário Curto do Adolescente (TEIQueASF), Questionário de regulação da emoção cognitiva (CERQ).

No estudo [E06], foi utilizado um sistema de tutoria de robô interativo, o qual consiste em um robô para atuar como agente de tutoria e um tablet para exibir o aplicativo de tutoria contendo problemas matemáticos a serem resolvidos pelo aluno. A aplicação foi 
feita apenas para uso nessa pesquisa. A avaliação foi conduzida por meio de questionário e da observação visual do professor pesquisador.

No trabalho [E09], foi utilizado o software Cara de Aprender (CADAP), que é um software desenvolvido para identificar as reações emocionais dos alunos através de suas expressões faciais durante a exposição de uma aula.

Em [E10], foi utilizado o robô COZMO e uma interface interativa pra aplicação das atividades, também foi aplicado o questionário de avaliação online criado por The Greater Good Science Center pra inferir a inteligência emocional dos alunos durante o processo de aprendizagem. No trabalho [E04] a computação foi utilizada por meio do Processo de Design Thinking aplicado em formato de framework em tarefas que envolviam a Robótica.

Em [E06], desenvolveu-se uma metodologia na qual as crianças interagiam com um robô (projetado pela equipe) por meio de um aplicativo de tutoria, os alunos podiam pedir ajuda ao robô por meio de botões na tela do tablet. O sistema de tutoria fornecia dicas de resolução de acordo com a necessidade do aluno. Em [E07], foi implementado um programa de instrução com quatro etapas: identificação do problema, modelagem do mecanismo, simulação e prototipagem.

Por fim, no trabalho [E08], o processo de verificação da regulação emocional aconteceu durante a execução de um curso preparatório de algoritmos para turmas do curso de ciência da computação. A Computação esteve presente somente no uso dos computadores durante o curso, sendo necessários na utilização durante as aulas. Não foi evidênciado pelos autores o uso de softwares específicos.

\section{Considerações Finais}

O objetivo deste MSL foi investigar estudos que demonstrassem em seu desenvolvimento metodologias, técnicas, softwares ou outras formas de utilização da computação no processo de regulação emocional de alunos da educação básica ao ensino superior. A busca se caracterizou por identificar abordagens que envolvessem o Pensamento Computacional aliado ao ensino de disciplinas da grade curricular dos alunos, com o próposito de identificar como os educadores vêm utilizando a computação no ambiente de ensino, visando catalogar e identificar fatores positivos e negativos dessas metodologias.

Outro ponto de interesse dessa pesquisa foi desvendar como foi realizado o processo de detecção ou estímulo da regulação emocional dos alunos durante as atividades desenvolvidas, visando mapear documentos como questionários, formulários ou demais artefatos que se fizeram úteis nesse processo, assim como quais emoções estiveram envolvidas nos estudos e como os pesquisadores as identificaram.

Entre os dez estudos primários, somente um deixou evidenciado de forma clara o uso do Pensamento Computacional no processo de regulação emocioal dos alunos, portanto, fica ainda mais nítido uma necessidade de investigação e desenvolvimento de estudos que possam relacionar conteúdos do Pensamento Computacional aliados no processo de regulação emocional de alunos em ambiente escolar.

Como trabalhos futuros, é proposto a investigação comparativa entre as ferramentas detectadas nessa pesquisa para a construção de uma aplicação capaz de realizar o estímulo da regulação emocional no processo de ensino, também fica como proposta para 
novos trabalhos a construção de um artefato que possa ser utilizado como meio de coleta de dados sobre os aspectos emocionais dos alunos.

\section{Referências}

Abed, A. L. Z. (2016). O desenvolvimento das habilidades socioemocionais como caminho para a aprendizagem e o sucesso escolar de alunos da educação básica. Construção psicopedagógica, 24(25):8-27.

Cabrejos, L. J. E. R., Viana, D., and dos Santos, R. P. (2018). Planejamento e execuçao de estudos secundários em informática na educaçao: Um guia prático baseado em experiências. Jornada de Atualização em Informática na Educação, 7(1):21-52.

CIEB (2021). Currículo de referência em tecnologia e computação (crtc). https://curriculo.cieb.net.br/. online; acessado em fevereiro 2021. Technical report.

Ekman, P. and Friesen, W. V. (2003). Unmasking the face: A guide to recognizing emotions from facial clues, volume 10. Ishk.

Fonseca, V. d. (2016). Importância das emoções na aprendizagem: uma abordagem neuropsicopedagógica. Revista Psicopedagogia, 33(102):365-384.

Keele, S. et al. (2007). Guidelines for performing systematic literature reviews in software engineering. Technical report, Citeseer.

Lima, T., Barradas Filho, A., Barros, A. K., Viana, D., Junior, J. B. B., and Rivero, L. (2020). Avaliando um jogo educacional para o ensino de inteligência artificial-qual metodologia para avaliação escolher? In Anais do XXVIII Workshop sobre Educação em Computação, pages 66-70. SBC.

Lopes, A. F., Santana, T. S., and Braga, A. H. (2020). O ensino de pensamento computacional por meio de jogos desplugados e olimpíadas científicas: um relato de experiência nos anos finais do ensino fundamental. In Anais do XXVIII Workshop sobre Educação em Computação, pages 96-100. SBC.

Moher, D., Stewart, L., and Shekelle, P. (2015). All in the family: systematic reviews, rapid reviews, scoping reviews, realist reviews, and more. Systematic reviews, 4(1):12.

Paviani, O., Adriano, D. D., and Wangham, M. S. (2018). Revisão sistemática da literatura sobre autenticação anônima em redes veiculares. Anais do Computer on the Beach, pages $170-179$.

Rodrigues, M. (2015). Educação emocional positiva: saber lidar com as emoções é uma importante lição. Novo Hamburgo: Sinopsys.

SBC (2018). Diretrizes para ensino de computação na educação básica. https://sbc.org.br/documentos-da-sbc/send/131-curriculos-de-referencia/1177diretrizes-para-ensino-de-computacao-na-educacao-basica. online; acessado em março 2021. Technical report.

Tessaro, F. and Lampert, C. D. T. (2019). Desenvolvimento da inteligência emocional na escola: relato de experiência. Psicol. esc. educ, pages e178696-e178696. 